\title{
Erratum
}

\section{Stabilization of cluster dimers by centrifugal effects}

\section{F. Calvo ${ }^{1}$, F. Spiegelmann ${ }^{2}$}

1 Laboratoire Collisions, Agrégats, Réactivité (UMR 5589, CNRS), IRSAMC, Université Paul Sabatier, 118 Route de Narbonne, F-31062 Toulouse Cédex, France

2 Laboratoire de Physique Quantique (UMR 5626, CNRS), IRSAMC, Université Paul Sabatier, 118, Route de Narbonne, F-31062 Toulouse Cédex, France

Z. Phys. D 41, 195-203 (1997)

In our recent article on cluster dimers [Z. Phys. D 41, 195-203 (1997)], two minor mistakes can be found.

1. In Eq. (18), the reduced mass $\mu^{*}$ should obviously be defined by $\left(\mu^{*}\right)^{-1}=m^{-1}+[(N-1) m]^{-1}$.

2. In Figs. 3c and $4 \mathrm{c}$, the labels $T$ and $\delta$ have been inverted. As mentioned in the captions, the solid lines are for the short-time rovibrational kinetic energy $T$, while the dashed lines are for the short-time rms bond length fluctuation $\delta$. The corrected figures follow.

(c)

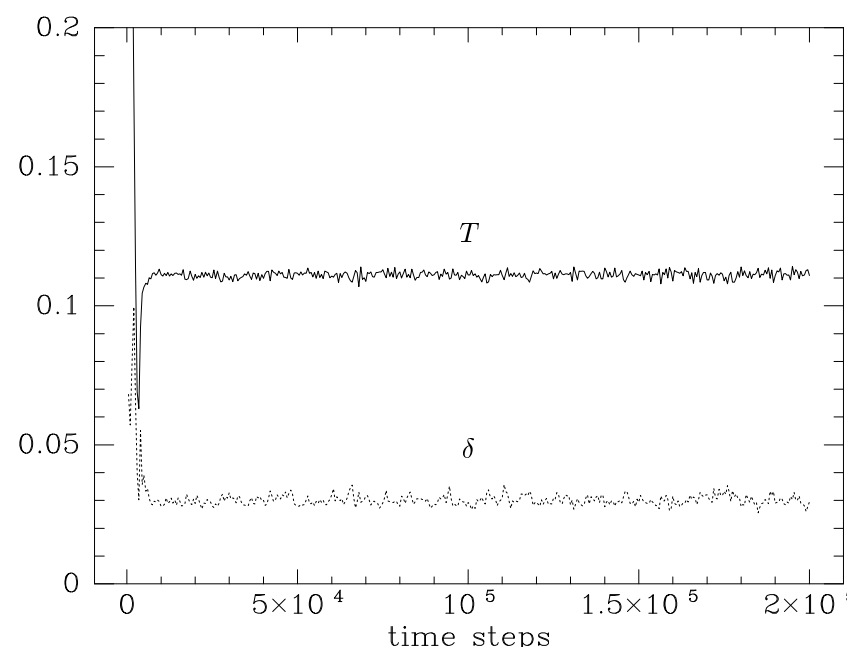

Fig. 3c. Simulation of a collision with characteristics $L / N=1.7$ and $E_{c} / N=0.476 \mathrm{LJ}$ units. c) Short-time rovibrational kinetic energy (solid line) and short-time rms bond length fluctuation $\delta$ (dashed line) as function of time. All units are LJ, $\delta$ has no unit (c)

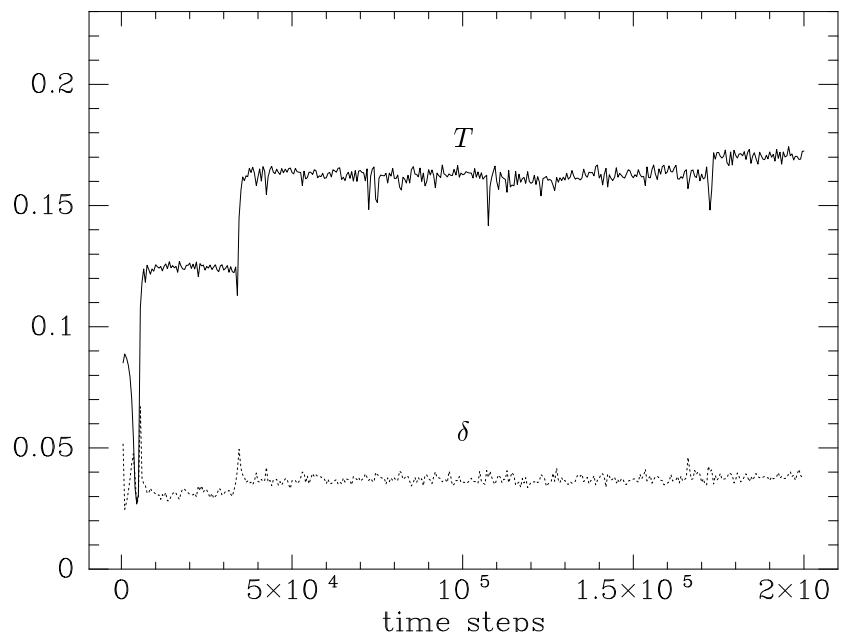

Fig. 4c. Simulation with characteristics $L / N=1.0$ and $E_{c} / N=0.120 \mathrm{LJ}$ units. c) is the same as Fig. 3c 